\title{
Towards "Post-Digital". A Media Theory to Re-Think the Digital Revolution
}

\section{Francesco Striano}

ORCID: https://orcid.org/0000-0003-3376-2485

(North-West Italy Philosophy Consortium, University of Turin, striano.francesco@yahoo.com)

\section{What Is Meant By "Post-Digital”?}

The first question is the following: are we currently in a post-digital condition? The answer may differ, depending on the definition of "post digital" that we intend to provide.

If we consider the post-digital condition as a situation in which digital has become part of everyday use and has become integrated into everyday life, action and gestures, then the answer may be yes. If we think of the omnipresence of digital devices and of their interfaces, or of the incorporation of digital technologies into commonly used objects that communicate with each other, even without the need of a human component (Internet of Things), then it must certainly be admitted that the above mentioned condition is already present.

In this case the advent of post-digital should not even be considered a recent achievement: Nicholas Negroponte, already in 1995, said that «[c]omputing is not about computers any more. It is about living» (Negroponte 1995, 6).

This vision agrees with the theory of the phases of affirmation of a new technology elaborated by Marshall McLuhan in his Laws of Media. According to the great Canadian scholar, there is a first phase in which the new technology itself is subject to attention and avant-garde experimentation, followed by a second phase in which the new technology becomes a simple commodity and the focus shifts from the medium message to the ubiquitous content (McLuhan \& McLuhan 1988). This second and last is the phase of cultural accommodation, the phase in which the new technology become a simple technology. Is the "post-new" phase.

However, there is another view that proposes that the term post-digital should refer to a critical reflection on digital (Andersen, Cox, \& Papadopoulos 2014), to a full awareness of the influence of digital culture and technologies on our modes of perception, 
cognition and action. The fact that digital has become part of our everyday life does not mean that contemporary culture has properly "digested" the supposed "digital invasion" of our cultural world.

We live in a mass digital culture, not in a post-digital one: digital media is ubiquitous, widespread, accessible, but it is often not well understood. The so-called digital natives, born already immersed in a digital broth culture, have now internalized the digital "gesture", but not the rules and the awareness of the logic inherent in the technologies they use.

If we consider that a more aware class of users is a goal to pursue, in order to avoid risks related to the reckless use of new technologies, then we must understand post-digital as an aim to be achieved. This aim splits into two directions: the first is about preparing the right means so that passive acceptance and the internalization of digital gestures translate into a full understanding of the functioning of media and of the operations they perform ${ }^{1}$; the second concerns ensuring that better knowledge of the processes gives the opportunity to really act on them, transforming the simple users into post-digital actors.

The task of a philosophy of digital, therefore, must be to critically and epistemologically rethink the digital, thus providing the tools to fully understand it. However, to supply philosophy with a base on which to speculate, a clarification of the subject of study is needed: a good media theory is needed.

\section{Re-Thinking the Digital: The Role of Media Theory}

The role of a good media theory in the contemporary condition must be to prepare the ground for a technically and technologically informed philosophy. This is because, if philosophy has the ambition to face the important challenges posed by digital technologies to our ways of constructing ourselves as human beings, then it cannot disregard a clear definition of what digital is.

Media theory, therefore, should be characterized as an analytical discipline, aimed at clarifying and dissolving problematic issues. The first thing that should be clarified is the definition of "digital" itself. In fact, it will be necessary to overcome some prejudices related to the concept of digital (i.e. digital as a synonym of "technologically advanced"; digital as "instantaneous" or "immediate"; digital as "immaterial" or "software"), as well as to analyze it in relation to the recording, transmission and processing of data.

In short, in order to transform our condition into the digital environment, we first need a theoretical clarification, which is articulated - at least initially - in three parts.

1 The awareness towards digital is not acquired without effort since, given the difference in speed in human and computational performance, to the machine it is better to bypass human understanding, so as to "train" the human component to its own rhythms. 


\subsection{Discrete vs. continuous.}

Often, in common perception, digital is conceived as something technologically advanced, eminently new and radically opposed to analog. To understand how much truth or error there is in this, we must clearly define what we mean by digital: "digital" means, first of all, "discrete". A digitization process is first and foremost discretization and sampling process.

Digital as a discretization of continuous flows and as a mathematization of natural phenomena has very ancient precedents. In a sense, different media, from the oldest to some newer ones, have been digital: from counting with fingers (digita means "fingers" in Latin) to the Morse code, passing through the vocal alphabet that discretizes the continuity of sound. Digital technologies have, from the beginning, served to take a distance from natural phenomena (Ong 2012, 80-81), to circumscribe them and make them reproducible. Without more advanced technologies, it was extremely difficult to analogically reproduce the continuous flows that characterize our experience of reality; for this reason the ancients already used discrete units to externalize language, or to set mathematical calculations on supports. Precisely those early digital technologies, the distance taking, the externalization, have influenced the way of thinking of human beings, giving rise to the development of new cultural forms.

Analog media, on the other hand, are those that simulate - and are therefore presented as analogues of the external world - or those that translate a continuous quantity into another (always continuous) directly proportional to it. Photography, phonography, electro-magnetic broadcast media (radio, television) are all examples of analog media in which physical reality writes directly on a material support, which is able to reproduce its continuous flow. The advantage of analog media is their fidelity (for better or for worse: they reproduce what is recorded, but without distinguishing between message and noise). However, unlike digital technologies, it is more difficult, with analog ones, to manipulate the collected data; in fact, for that purpose, it is convenient to electronically sample analog signals and transform them into digital ones.

In the computer, the digital "returns" and makes the frequency of the signal, modulated - according to a binary logic - in discrete pulses, the heart of its operation. «[I] n techno-mathematical terms, the numerical ("digital") signal analysis in the frequency domain is the inverse value ("Kehrwert") of the analogue wave form in the time domain»" (Ernst 2015).

As already mentioned, contemporary digital technologies are not only based on a process of discretization and mathematization, but also of binarization. This is in order to increase the amount of information that can be stored and processed by a system. The unit of information measurement (bit) is defined as the logarithm to the base two of the

2 This present essay is largely indebted to Wolfgang Ernst, to my reading of his texts, to his seminars, and to private conversations. 
possibilities of choosing among possible alternatives, being 1 bit $=\log 2$ (two choices, equally possible). The amount of information can be calculated as minus the sum of the multiplication of the probability value of each alternative for the logarithm to the base two of the same. This tells us that information capacity can be increased by increasing the number of choices and is greater if these choices are equally probable. Increasing the number of choices possible in a stochastic system, however, increases the degree of unpredictability and randomness. A way for increasing information capacity, but with a good degree of predictability is to connect circuits that work each with two possible values (Shannon \& Weaver 1964, 8-16).

What we call "digital" today, referring to computers and devices derived from it, is discrete, electronic, mathematical, binary and algorithmic. Each of these elements has its own theoretical and ontological implications, which cannot be detailed here, but which, once revealed by media theory, must be taken into due consideration by the philosophy of information and by any reflection on the contemporary digital condition.

The one just presented does not want to be a rigid historiographical division: digitalanalog-digital. Often certain elements are co-present and analog and digital are ways of representing and processing the same physical quantities. Moreover, from the standpoint of a non-linear media history, it must be recognized that the vacuum tube (in particular, the thermionic valve) is at the origin of the development of analog media such as radio and television, as well as of the digital medium par excellence, that is the computer. Finally, there are also hybrid media: cinema on film, for example, although based on analog photography technology, gives an illusion of movement through the rapid succession of discrete frames; even in digital photography, before the mathematization of the image, an essentially analog process takes place, i.e. that for which the stronger the light that hits a single photosite (a small portion of a semiconductor element that constitutes the surface of a sensor like the CCD), the more electrons will gather on it, just as, on a photosensitive film, more silver bromide atoms will clump in the areas hardest hit by light (Mara 2006, $53)$.

A media theory working on these issues will first show us how "digital" is not synonymous with "technologically advanced" or "analog" with "obsolete".

\subsection{Time and temporality.}

Another task of media theory, in its clarifying function on the nature of digital, must be to connect the difference between analog and digital to the discourse on temporality. In fact, the difference between discrete and continuous also - and above all - regards the temporal dimension. Furthermore, the measurement and modulation in electronic media concerns the transmission of information through a variable signal in a random manner over time, to be considered either in its continuity (analog: time-continuous signal), or to be sampled in discrete units (digital: time-discrete impulse).

Beyond the discord on the "nature" of time, the fact that it could be measured either 
continuously or discreetly is an ancient idea. It may seem risky, but it is not amiss to resort to Aristotle in order to explain the difference between analog and digital. According to the Stagirite, time as such, sinceit is in relation to the continuous movement, is itself continuous. But time as counting (by numbers, or "digits"), delimited by instants through which "first" and "then" are defined, is discreet (Aristotle 1936, 385/ss).

Of opposite opinion regarding reality, but of similar views regarding the double representation of time, was Leibniz. From his point of view reality is discrete, as it is formed by monads (discrete and inextensive). Space and time are ideal (resulting from the human perspective), so they are potential and not actual. In what is ideal, the whole precedes the parts, so time (as well as space) is essentially continuous. Nevertheless, it can be represented discontinuously as a succession of instants, because through the idea of time we set in succession real discrete events (Leibniz \& Clarke 2000, 36ff).

What happens in the technological media is that these different conceptions or representations of time can be materially implemented. The difference between live (in analog media) and real time (in digital media) is particularly informative in this regard. Analog media are characterized by live broadcasting, namely the very "live" transmission of a continuous electro-magnetic modulate signal. Digital communication, instead, happens in real-time, that is a process of constant micro-archiving and re-presentification of the data collected and transmitted through an algorithmic process: it is a non-instantaneous transmission and, however fast, intrinsically delayed. The present in real time doesn't exist: the transmission is in fact already outdated and contains both the redundancy of what preceded it, as well as an anticipation of what will happen after, in a mechanism of retention and protention ${ }^{3}$ technically (re)produced (Ernst 2017, 25ff).

Temporality must be one of the main fields of investigation of a good media theory precisely because contemporary media are inherently temporal. Analogue media are undoubtedly time-based, since «they have duration as a dimension and unfold to the viewer over time» (Dover 2014). But digital media could be termed time-critical, since not only the duration in the linear sense, but the temporality in the broader sense is part of their "essence". The temporal processes take place in the machine: if, for example, in writing a code, the different operations can coexist on the same plane, in their implementation in the machine they become a sequence of (discontinuous) signals over time (Ernst 2004, 19-20).

In digital media every operation must be instantiated at execution time and this time is regulated within the machine itself and is technologically implemented. An example is the integrated circuit 555: it is a timer, or clock, even if this name is improper. It does not measure time, but rather it gives time. 


\subsection{The hardware oblivion.}

A common prejudice in mass digital culture, which media theory should contribute to eradicate, is that according to which analog has something to do with hardware, while digital is about software. In reality, the digitization process is already at the hardware level where a continuous signal is discretized (in a binary structure) through a series of resistances.

The same logic on which the codes and programming languages are based is already incorporated in the hardware. Shannon, whose mathematical theory of communication (Shannon \& Weaver 1964) has made possible the evolution of the current technological media, has also laid the foundations for the concrete implementation of the Universal Turing Machine: in his 1938 essay, in fact, he worked on developing a general theory of circuit design, translating Boolean logic into functional schemes ready to be constructed and used to carry out any logical operation, regardless of the content (Shannon 1938, 3880).

This point is not without consequences from a philosophical view, since it leads to a purely techno-materialistic ontology. It is a fact sometimes ignored even by a more cultural-oriented media theory; a rigorous analytical media theory, however, brings to light this important repression, that is the indispensability of the hardware, not only as support, but as a condition of possibility and as an introduction of logic into matter.

This ontological assumption can lead us to treat software as an emergency - that is, a superior property that springs from hardware and cannot exist without it - or, more radically, as a mere epiphenomenon - a phenomenological appearance that adapts to human perception, but that simply represents what happens at the hardware level ${ }^{4}$. A standard bearer of the second hypothesis was Friedrich A. Kittler. In his words: «Software does not exist as a machine-independent faculty», so there are "good grounds to assume the indispensability and, consequently, the priority of hardware in general»; and that's the reason why, strictly technically speaking, there is no software, or, even if it existed, it «would just be a billion dollar deal based on the cheapest elements on earth» (Kittler 1995).

In any case, whatever position one wants to take, connecting two elements captured by a serious media-theoretical analysis, one will have to reach an important conclusion on the nature of the digital: if (i) digital is a way of (spatially and temporally) representing reality, and if (ii) software is strictly dependent on hardware, then we must conclude that the digital environment is not a "virtual" world somehow separated from the "real" world.

Also this statement, obtained through a rigorous analysis of the technical-media apparatus, provides not negligible philosophical insights: our ontology is considerably

4 Even cloud computing, while using the "cloud" metaphor, cannot be separated from its hardware base. The joke "there is no cloud, it's just someone else's computer" refers to the fact that what we store in cloud services, is, in fact, materially stored in remote servers, often owned by large data companies such as Google. 
simplified, digital objects are subtracted both from a metaphysics of virtual reality and from being treated as mere communicative phenomena, while, from an ethical point of view, a real responsibility can be established for actions carried out, for instance, online (Striano 2018, 92-106).

\section{Problems in Digital Communication}

As mentioned above, an ontological clarification on the nature of digital media necessarily leads to ethical effects. Moreover, as will be shown, a better theoretical understanding of digital can also lead to a more correct definition of the framework of the ethical problems connected to the CMC.

First of all, the main themes need to be summarized, normally addressed by ethics of communication, which should be brought under the aegis of a philosophy of digital in order to be studied profitably. The main problems that are observed are: the stiffening of identity and of beliefs, the misrecognition of the otherness, and the reduction of empathic skills (Turkle 2015). If we take into account the peculiar digital ontology we tried to lay the foundations for, then the solution to this kind of problems cannot be found through an "ethic of communication" that ignores the technical specificities of the computermediated communication (CMC). At the same time, since virtual environment is not a completely separate social context, not even an "ethic in communication" can help us: neither censorship - however always difficult to practice on social networks in which people sometimes create even more than one account, just to circumvent the ban - or the appeal to a netiquette - since its violation is a systematic fact, connected to the structure of the IT media - will have the desired effects (Striano 2018, 105).

The proposal that I intend to advance in this essay is to identify two possible causes of these ethical problems, and then to suggest a theoretical approach in order to formulate a more complete information ethics possible.

The first of the causes strictly connects the communication problems with the technical ones: the emergence of violent behavior or closure is not only connected to the violation of communication standards, but also to the fact that too often we forget the signifiers. Mass digital culture is a paradoxical condition of ease in an environment saturated in digital media, often ignoring their mechanisms and functioning. In this condition, we are driven to shift our attention from the signifiers (that, in fact, guide and condition our way of understanding, acting and communicating ${ }^{5}$ ) to the exclusively semiotic field, that of meanings. In this case, the signifiers are those means and material supports through which digital communication takes place: in other words, digital media.

In relation to this issue, we see how a good media theory is a necessary

5 This is a position that could be called a techno-determinist one, according to which «media determine our situation» (Kittler 1999, p. xxxix, italic mine). But even without being so radical, we can embrace a theory that media condition courses of action and our position in such courses. 
presupposition to re-emerge the importance of signifiers and to remedy this oblivion. To do so, media theory to which I refer should have some precise characteristics: research of the technological a priori that make certain phenomena possible, study of time-critical aspects, attention to hardware, reverse engineering. Essentially, the media theory that provides the ideal analytical basis on which to engage an informed speculation on the digital, is media archaeology, and, in particular, radical media archaeology (Ernst 2018, 35-43).

The second cause is what I call "decline in hermeneutic attention": because of the speed of communication it is easy to lose the sense of the frame and, consequently, the impression of immediacy replaces the awareness of mediation; less attention is given to mediated messages that would require careful interpretation. This is room for philosophy and concerns the possibility of applying hermeneutics to CMC, characterized, as we have seen, by a rigidly logical and binary basis and by an imperative language.

\section{Conclusion}

As will now be understood, the intent of this essay is not to delve into the philosophical questions just mentioned, but rather to highlight them. This is a preliminary work, aimed at bringing out the importance and necessity of a good media theory that serves as a precondition for an informed philosophy of information and of digital.

This paper started from a definition of post-digital as a state of awareness and exploitation of the digital condition, considering it desirable and largely preferable to the current mass digital culture. The assumption is that problems and risks that are attributed by some to the digital condition, are actually due to the lack of understanding of the aforementioned condition and of its consequences.

To achieve this state it is appropriate to have a philosophical work that, contaminated by technical and scientific knowledge, inserts the element of criticism and understanding in the analysis of the digital. To be able to carry out this work, however, an analytical undertaking is necessary, which clarifies the object of analysis as best as possible. This is why media theory is indispensable, and it must have the tools to deal with issues such as the difference between analog and digital in terms of space and time. Not only that: it must remedy the oblivion of the hardware, showing how, in the contemporary digital condition, the mathematical, binary, algorithmic logic is already incorporated in the matter. This is not a secondary task, because this clarification allows us to clear away speculations that consider digital (or virtual) as a world separate from so-called reality, thus failing to satisfactorily explain the interaction between the two. Media theory that addresses these problems and offers the most convincing explanations is media archaeology. I therefore propose to embrace its most radical version and the resulting techno-materialist ontology.

However I think it is not enough to stop at the analytical part of the path of understanding, in order to transit in a post-digital condition. We need to find an effective 
synthesis, which shows how the acquisitions of media theory can help us avoid risks of disorientation in the digital environment. We need a philosophy that metabolizes and guides the transition.

We need a philosophy that reflects on the concepts of media and mediation without ignoring the technical aspects. This philosophy must also have a public function that consists in bringing out the hidden elements, yet bearing, of media that inform the entire public life (in our time, digital media). An option - that could be the starting point of this philosophy - could be the Simondonian concept of general technology. According to Simondon, the human individual is involved in the individuation of the technical object. Therefore there are functional schemes that link the two individualities and «a technique of all techniques can be developed by the generalization of schemes» (Simondon 2012, 298 - my transl.). The discovery of these schemes is defined as general technology and would allow us to find a fundamental unity between technicality and political thought in the reconstruction of a reticular structure different but similar to that of original magical thought.

In order to be able to assume its public function, general technology must pass from academic discussion to general culture and must be able to influence public debate and consequent policies. It will have to incorporate the analysis of algorithms underlying computation, the understanding of the micro-archival temporal regime, and the understanding of the physical nature of bits and of digital phenomena rooted in the continuous analog, as well as a call for technical and media education since the very first years of school, for ethics of communication and ethics new technologies, and for hermeneutic education.

Once the elements that make up the digital technological apparatus and which decisively influence contemporary culture have been clarified, they will be elaborated and made widespread knowledge. Only then can we finally become post-digital.

\section{Literature}

Andersen C. U., Cox G., \& Papadopoulos G. 2014. "Editorial” in APRJA Post-Digital Research 3(1).

Aristotle. 1936. Physics, ed. by W. D. Ross. Oxford: Oxford University Press.

Dover C. 2014. "What Is "Time-Based Media”?: A Q\&A with Guggenheim Conservator Joanna Phillips," The Guggenheim Museums and Foundation Web Page, last modified 4 March, 2019, accessed 29 March, 2019, https://www.guggenheim. org/blogs/checklist/what-is-time-based-media-a-q-and-a-with-guggenheimconservator-joanna-phillips 
Ernst W. 2004. Medienwissen(schaft) zeitkritisch. Ein Programm aus der Sophienstraße. Berlin: Humboldt Universität.

Ernst W. 2015. “Posting” Digital Presence. A Micro-Temporal Regime.” Keynote Lecture at BFX Academic Conference Analogue to Post-Digital, Bournemouth University, September 25th-26th 2015.

Ernst W. 2017. The Delayed Present: Media-Induced Tempor(e)alities \& Techno-traumatic Irritations of "the Contemporary". Berlin: Sternberg Press.

ERnst W. 2018. "Radical Media Archaeology (Its Epistemology, Aesthetics and Case Studies)." Artnodes 21:35-43.

Husserl E. 1991. On the Phenomenology of the Consciousness of Internal Time. Trans. J. B. Brough. Dordrecht - Boston - London: Kluwer Academic Publishers.

Kittler F. A. 1995. “There Is No Software.” CTheory 10/18/1995.

Kittler F. A. 1999. Gramophone, Film, Typewriter. Trans. by G. Winthrop-Young \& M. Wutz. Stanford: Stanford University Press.

Leibniz G. W. \& Clarke S. 2000. Correspondence, ed. by R. Ariew. Indianapolis Cambridge: Hackett.

Marra C. 2006. L'immagine infedele. La falsa rivoluzione della fotografia digitale. Milano: Bruno Mondadori.

McLuhan M. \& McLuhan E. 1988. Laws of Media. The New Science. Toronto: University of Toronto Press.

Negroponte N. 1995. Being Digital. Toronto - New York: Random House.

Ong W. J. 2012. Orality and Literacy: The Technologizing of the World (30th Anniversary Edition). New York: Routledge.

Shannon C. E. 1964. "A Symbolic Analysis of Relay and Switching Circuits." Transactions American Institute of Electrical Engineers 57(1938).

Shannon C. E. \& Weaver W. 1964. The Mathematical Theory of Communication. Urbana: The University of Illinois Press.

Simondon G. 2012. Du mode d'existence des objets techniques. Paris: Aubier.

Striano F. 2018. „Fenomenologia del cyber-stupro. Note ontologico-filosofiche sulla violenza informaticamente mediata." Lessico di Etica Pubblica 1/2018:92-106. 
Francesco Striano (Turin)

\title{
Towards “Post-Digital”.
}

\section{A Media Theory to Re-Think the Digital Revolution}

\begin{abstract}
Can we say we live in a post-digital condition? It depends. This paper sets out to distinguish between the current mass digital culture and an authentic post-digital culture.

If we mean "post-digital" as the full internalization and awareness of the result of the so-called digital revolution, then it is necessary a philosophical work to discuss related problems, identify the causes and propose solutions.

An authentic philosophy of digital will, however, have to start from a clarification of the terms and basic objects of its investigation. Here media theory is inserted as an analytical tool: the purpose of this essay is to outline a road map for a good media theory that interfaces with questions of definition of digital, also in light of the notions of space, time, and matter. As will be seen, the description given here for a "good media theory" does, in fact, coincide with an already existing - and inserted in the contemporary debate - school. In conclusion we will try to delineate the field of philosophical inquiry opened by the clarification brought by the previous analysis, and to suggest a general framework within which philosophy will have to move in order to finally reach the authentic postdigital condition.
\end{abstract}

Keywords: CMC; digital; information; media; theory.

Ethics in Progress (ISSN 2084-9257). Vol. 10 (2019). No. 1, Art. \#7, pp. 83-93.

Creative Commons BY-SA 4.0

Doi:10.14746/eip.2019.1.7 